\title{
MRS Members Elect 1992 Officers, Councillors
}

In the yearly elections held this past September, MRS members elected eight colleagues to leadership positions in the MRS Council. Newly elected as MRS officers are First Vice President and President Elect, S. Thomas Picraux, Second Vice President John C. Bravman, and Secretary Lynn A. Boatner. The six newly elected Councillors include Aaron N. Bloch, R.P.H. Chang, Russell R. Chianelli, Robert Hull, Carol M. Jantzen, and Paul S. Peercy.

Previously elected First Vice President in 1991, G. Slade Cargill automatically becomes the 1992 MRS President. Also in 1992, Charles B. Duke continues as Treasurer in his two-year position, and 1991 President James B. Roberto serves as Immediate Past President.

\section{MRS Officers}

President G. Slade Cargill III (1992)

IBM T.J. Watson Research Center

Immediate Past President

James B. Roberto (1992)

Oak Ridge National Laboratory
First Vice President (President Elect)

* S. Thomas Picraux (1992)

Sandia National Laboratories

Second Vice President

* John C. Bravman (1992)

Stanford University

Secretary

* Lynn A. Boatner (1993)

Oak Ridge National Laboratory

Treasurer

Charles B. Duke (1992)

Xerox Webster Research Center

1992 MRS Councillors

John E.E. Baglin (1992)

IBM Almaden Research Center

* Aaron N. Bloch (1994)

Columbia University

* R.P.H. Chang (1994)

Northwestern University

* Russell R. Chianelli (1994)

Exxon Research and Engineering

Company

Mildred S. Dresselhaus (1992)

Massachusetts Institute of Technology
Gregory C. Farrington (1993)

University of Pennsylvania

J. Murray Gibson (1992)

University of Illinois-Urbana

* Robert Hull (1994)

AT\&T Bell Laboratories

* Carol M. Jantzen (1994)

Westinghouse Savannah River

Company

Edward J. Kramer (1992)

Cornell University

* Paul S. Peercy (1994)

Sandia National Laboratories

Julia M. Phillips (1993)

AT\&T Bell Laboratories

Rustum Roy (1993)

Pennsylvania State University

Frans Spaepen (1992)

Harvard University

Carl V. Thompson (1993)

Massachusetts Institute of Technology

* Newly Elected

(Tern of office expins at end of year indionted in parnthesis.)

\section{MRS Establishes David Turnbull Lectureship}

The Materials Research Society recently approved establishment of the David Turnbull Lectureship. This yearly award will recognize the career of a scientist who has made outstanding contributions to understanding materials phenomena and properties through research, writing, and lecturing, as exemplified by the life work of David Turnbull. The Lectureship will also be a means to provide lectures of exceptional quality and scientific significance for the MRS Fall Meeting as well as, possibly, MRS Section and University Chapter meetings.

The Turnbull Lecturer will receive a $\$ 2,000$ honorarium and a citation plaque. The award will include travel expenses to the MRS meeting at which the Turnbull Lecture is given and also additional funds to enable the recipient to speak as the Turnbull Lecturer for MRS Sections and University Chapters.

Plans are to name the first David Turnbull Lecturer at the 1992 MRS Fall Meeting and nominations are being accepted.

\section{Rules and Eligibility}

Nominations for the Turnbull Lecturer may be made for scientists and engineers in all areas of materials science. The primary consideration in making the award is the nominee's career contribution to the fundamental understanding of materials phenomena and properties, through research, writing, and lecturing.

Nominees need not be members of the Materials Research Society, and nominees of any national origin or citizenship are eligible.

Current members of the MRS Awards Committee, MRS officers, and previous Turnbull Lecturers are not elibible.

Selection of the Turnbull Lecturer must be approved by the MRS Executive Committee with recommendation from the Awards Committee. The decision of the Executive Committee is final.

The Lectureship will not be awarded in absentia. The Lecture must be given within 12 months of the selection at an MRS Fall Meeting.

\section{Nomination Procedure}

A package containing the following is required:

1. A statement by the nominator supporting the candidate's suitability to be designated the Turnbull Lecturer, with re- spect to the importance of the candidate's research contributions to the fundamental understanding of materials, quality of the candidate's writing and lectures, candidate's role in education and graduate training in materials research.

2. Supporting information and documents, e.g., curriculum vitae including a list of key publications.

3. Letters of support from established scientists familiar with the nominee's qualifications and area of research. Only three such letters will be accepted with each nomination. Each letter should make specific reference to the criteria listed in item 1 .

4. A list of supporting documents submitted. The entire nomination package, excluding ( $p$ )reprints, should not exceed 12 pages.

A nomination for a Turnbull Lectureship remains under consideration for three years, and it may be updated by the nominator during that time.

Nominations can be submitted to: John B. Ballance, Executive Director, Attention: Turnbull Lectureship (B), Materials Research Society, 9800 McKnight Road, Pittsburgh, PA 15237. 


\section{ITFEA}

The

DMDP 2000

SPECTROMETER

\section{“An enabling technology for Raman and Brillouin Spectroscopy"}

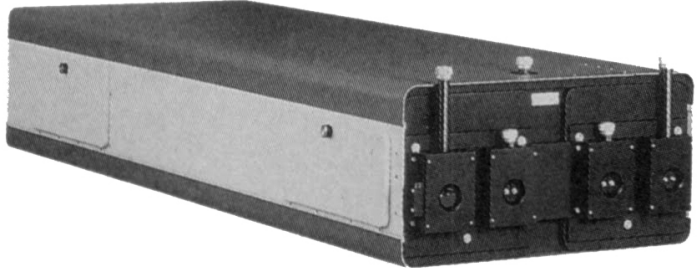

SOPRA's new DMDP 2000 high resolution spectrometer is a high performance two meter double-pass double monochromator.

The DMDP 2000 combines a resolution of $0.025 \mathrm{~cm}^{-1}$ and a contrast of $10^{-14}$ while utilizing the same sound engineering design for which SOPRA spectrometers are renowned. The resulting precision, flexibility, and stability truly put the DMDP 2000 in a class of its own.

The benefits of the DMDP 2000 can easily be recognised in a wide variety of spectroscopic techniques :

- Raman and micro-Raman spectroscopy

- Brillouin spectroscopy/acoustic mode analysis

- Fluorescence spectroscopy

- Emission spectroscopy

The DMDP 2000 is an enabling technology for the understanding of materials such as semiconductors, superlarties, HTC superoonductors and diamond.

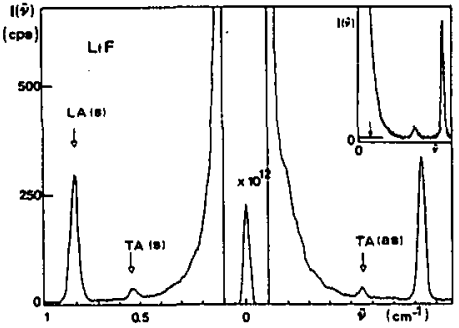

In order to get more information on the DMDP 2000, just fax or call :

North America

SOPRA Inc. : Tel. : (508) 263-25 20 - Fax : (508) 263-27 90

Japan

SEIKA Corp. : Tel. : (33) 346-83 11 - Fax : (33) 346-8314

Europe

SOPRA GmbH : Tel. : (06152) 5092 - Fax : (06152) 55201

SOPRA S.A. : Tel. : 47810949 - Fax : 42422934

Circle No. 40 on Reader Service Card.

\section{NEW FROM MRS-1991 Spring Meeting Proceedings}

Amorphous Silicon Technology - 1991

Editors: A. Madan, Y. Hamakawa, M. Thompson,

P.C. Taylor, P.G. LeComber, 1991, 885 pgs.

ISBN: 1-55899-113-1

Silicon Molecular Beam Epitaxy

Editors: J.C. Bean, S. lyer, K. Wang, 1991, 649 pgs.

ISBN: 1-55899-114-X Volume 220B

$\$ 52$ MRS Members $\$ 59$ U.S. List $\$ 66$ Foreign

Heteroepitaxy of Dissimilar Materials

Editors: R.F.C. Farrow, J.P. Harbison, P.S. Peercy,

A. Zangwill, 1991, 499 pgs.

ISBN: 1-55899-115-8 Volume 221B

$\$ 56$ MRS Members \$63U.S. List \$68 Foreign

Atomic Layer Growth and Processing

Editors: T.F. Kuech, P.D. Dapkus, Y. Aoyagi, 1991,

359 pgs.

ISBN: 1-55899-116-6 Volume 222B

$\$ 52$ MRS Members \$59 U.S. List \$66 Foreign

Low Energy Ion Beam and Plasma Modification of

Materials

Editors: J.M.E. Harper, K. Miyake, J.R. McNeil,

S.M. Gorbatkin, 1991, 397 pgs.

ISBN: 1-55899-117-4

Volume 223B

$\$ 49$ MRS Members \$56 U.S. List \$63 Foreign

Rapid Thermal and Integrated Processing

Editors: J.C. Gelpey, M.L. Green, J. Wortman,

R. Singh, 1991,503 pgs.

ISBN: $1-55899-118-2$

Volume 224B

$\$ 53.00$ MRS Members $\$ 60$ U.S. List $\$ 67$ Foreign
Materiais Reliability Issues in Microelectronics Editors: J.R. Lloyd, P.S. Ho, C.T. Sah, F. Yost, 1991, 359 pgs.

ISBN: 1-55899-119-0 Volume 225B

\$47 MRS Members \$54 U.S. List \$61 Foreign

Mechanical Behavior of Materials and Structures

in Microelectronics

Editors: E. Suhir, R.C. Cammarata, D.D.L. Chung,

M. Jono, 1991, 454 pgs.

ISBN: 1-55899-120-4 Volume 226B

$\$ 49$ MRS Members \$56 U.S. List \$63 Foreign

Materials Science of High Temperature Polymers

for Microelectronics

Editors: D.T. Grubb, I. Mita, D.Y. Yoon, 1991, 400 pgs.

ISBN: 1-55899-121-2 Volume 227B

$\$ 52$ MRS Members \$59 U.S. List \$64 Foreign

Materials for Optical Information Processing

Editors: C. Warde, J. Stamatoff, W. Wang

ISBN: $1-55899-122-0$ Volume 2288

$\$ 47$ MRS Members \$54 U.S. List \$61 Foreign

Structure/Property Relationships for Metal/Metal Interfaces

Editors: A.D. Romig, D.E. Fowler, P.D. Bristowe, 1991

357 pgs.

ISBN: 1-55899-123-9 Volume 229B

$\$ 52$ MRS Members \$59 U.S. List \$63 Foreign
Phase Transformation Kinetics in Thin Films Editors: M. Chen, M. Thompson, A. Schwarz, M. Libera

ISBN: 1-55899-124-7 Volume 2308

$\$ 42$ MRS Members \$49 U.S. List \$56 Foreign

Magnetic Thin Films, Multilayers and Surtaces

Editor: S.S.P. Parkin

ISBN: 1-55899-125-5 Volume 231B

$\$ 47$ MRS Members \$54 U.S. List \$60 Foreign

Magnetic Materials: Microstructure and Properties

Editors: T. Suzuki, Y. Sugita, B.M. Clemens, K. Oúchi,

D.E. Laughlin, 1991, 347 pgs.

ISBN: 1-55899-126-3 Volume 232B

$\$ 47$ MRS Members \$54 U.S. List \$60 Foreign

Synthesis/Characterization and Novel

Applications of Molecular Sieve Materials

Editors: R.L. Bedard, T. Bein, M.E. Davis, J. Garces,

V.A. Maroni, G.D. Stucky, 1991, 303 pgs.

ISBN: 1-55899-127-1 Volume 233B

\$47 MRS Members \$54 U.S. List \$60 Foreign

Modem Perspectives on Thermoelectrics and

Related Materials

Editors: D.D. Allred, C. Vining, G. Slack, 1991

245 pgs.

ISBN: 1-55899-128-X Volume 234B

$\$ 52$ MRS Members \$59 U.S. List \$66 Foreign

Order from: Materials Research Society, 9800 McKnight Road, Pittsburgh, PA 15237; Phone (412) 367-3012, FAX (412) 367-4373.

In Europe, Africa and the Middle East: Clarke Associates-Europe LId., 13a Small Street. Bristol BS1 1DE. England, Phone 0272 268864; FAX: 0272226437 


\section{(As of November 1, 1991)}

Advanced Control Systems

Corporation

Advanced Energy Industries, Inc.

Advanced Materials Laboratory, Inc.

Aerospace Corporation

AET addax, Inc.

AG Associates

Air Products and Chemicals, Inc.

Aixtron $\mathrm{GmbH}$

Akzo America Inc.

Alcan International Limited

Alcoa

Alliance Analytical

Allied-Signal Inc.

American Cyanamid Company

Ames Laboratory

Amoco Corporation

Amoco Chemical Corporation

Amoco Oil Co.

Amoco Technology Company

Anatech Ltd.

Angstrom Sciences

APD Cryogenics Inc.

APL Engineered Materials, Inc.

Applied Materials, Inc.

Applied Science and Technology, Inc. (AST $\mathrm{XX}$ )

Argonne National Laboratory/IPNS

Atomic Energy of Canada Limited

AT\&T Bell Laboratories

Bandgap Technology Corporation

Barbeau-Hutchings Advertising, Inc.

Bausch and Lomb

Bell Communications Research, Inc.

Billiton Precursors B.V.

Bio-Rad Laboratories

Blake Industries, Inc.

The BOC Group

Bomem Inc.

BP America Research \& Development

British Telecom Research Labs

Brookhaven Instruments Corp.

Bruker Instruments Inc.

Butterworths Scientific Lto.

Cameca Instruments, Inc.

Center for Waste Minimization \& Management

Centorr Furnaces/Vacuum Industries

Coherent Laser Group

Collagen Corporation

Commonwealth Scientific Corporation

Computer Graphic Service

Conversion Technology Corporation

Corning Inc./Corning Glass Works

Crystallume

CVC Products, Inc.

David Sarnoff Research Center

Denton Vacuum Inc.

Digital Equipment Corporation

Digital Instruments, Inc.

Doty Scientific, inc.

The Dow Chemical Company

Dow Corning Corporation

E.1. duPont de Nemours \& Company

Eastman Kodak Company

Eaton Corporation

EG\&G Idaho, Inc.

EG\&G Instruments, Inc.

EG\&G Princeton Applied Research

Electric Power Research institute (EPRI)

ElectroScan Corporation

Elettrorava S.p.A.

Elsevier Science Publishers B.V.

Emcore Corporation

Energy and Environmental Research Center (EERC)
Engelhard Corporation

EPI Division Chorus Corporation

Epitaxial Products International, Lid.

Epitronics Corporation

Charles Evans \& Associates

Evans East, Inc.

Extrel FTMS

Exxon Basic Chemicals Technology

Exxon Production Research Company

Exxon Research \& Engineering Co.

FEl Company

E.A. Fischone Instruments Manufacturing

FleXus Incorporated

Ford Motor Company

Forest Products Laboratory

Fuji Electric Co., Itd.

Fuji Xerox Co., Ltd.

Fujitsu Ltd.

Furukawa Electric Yokohama R\&D Lab

Gatan, Inc.

Gelest Inc.

General Electric Corporation

General Electric Corp./Advanced Inorganic Materials Lab.

General Electric Corp./Aircraft Engines Division

General Electric NMR Instruments

General Motors Research Laboratories

Genetics Institute, Inc.

Geo-Centers, Inc.

Getty Conservation Institute

Goodfellow Corporation

Granville-Phillips Company

GTE Laboratories Inc.

Hewiett-Packard, NMD

High Voltage Engineering Europa B.V.

Hitachi Lto

Hitachi Scientific Instruments

Hoechst Celanese Research

$$
\text { Division }
$$

Howmedica

Hoya Optics, Inc.

Hughes Research Laboratories

Huntington Mechanical Laboratories, Inc.

IBM Corporation

ICI Polyurethanes

Imperial Chemical Industries

Implant Sciences Corporation

Innovative Technology, Inc.

INSPEC/IEE

Instron Corporation

Instruments S.A., Inc./Riber Division

Intel Corporation

Ion Tech, Inc.

Ionic Atlanta

Janis Research Company, Inc.

JCPDS - International Centre for Diffraction Data

JEOL U.S.A. Inc.

Johnson Controls, Inc.

Johnson \& Johnson

Johnson \& Johnson Orthopaedics, Inc.

Kennametal, Inc.

Kinzoku Giken Co., Ltd.

Kluwer Academic Publishers

Kobe Development Corporation

Kobe Steel, Lto.

Kogakugiken Co., Ltd.

Kopin Corporation

Kratos Analytical, Inc.

Kyocera Corporation

Lake Shore Cryotronics, Inc

Lam Research Corporation
Lambda Physik, Inc.

Lawrence Berkeley Laborator

Lawrence Livermore National Laboratory

Lehighton Electronics, Inc.

Kurt J. Lesker Company

Leybold Vacuum Products, Inc.

Link Analytical/Oxford Group

Los Alamos National Laboratory

Lumonics, Inc.

Martin Marietta Energy Systems, Inc.

Martin Marietta Laboratories

Matec Instruments, Inc.

Materials Research Corporation

Matheson Gas Products

Matsushita Electrical Industrial Co.

MCC (Microelectronics \& Computer Technology)

MDC Vacuum Products Corporation

Medisorb Technology International

MEMC Electronic Materials Inc.

Micromap Corp.

Mitsubishi Electric Corporation

Mitsubishi Kasei Corporation

MKS Instruments, Inc.

Mobay Corporation

Mobil Research \& Development Corporation

Molycorp, Inc. (a Unocal Company)

MR Semicon, Inc.

Nano Instruments, Inc

NASA Lewis Research Center

National Electrostatics Corporation

National Renewable Energy Laboratory (NREL)

National Semiconductor

NEC Corporation

NEC Research institute Inc

Nippon Mining Company, Ltd.

Nippon Telegraph \& Telephone Corporation

Nissei Sangyo America, Ltd.

Nissen Electric Co., Lid.

Nisshin Flour Milling Co., Lid.

NKK Corporation

Noran Instruments, Inc.

North Eastern Analytical Corporation

Northern Telecom Electronics Ltd.

Norton Company

Oak Ridge National Laboratory

OIS, inc. (Optical Imaging Systems, Inc.)

Oxford Instruments North America Inc.

Oxford Plasma Technology

Pacific Northwest Laboratory

Peak Systems, Inc.

Pechiney Corporation

Pergamon Press, Inc.

Perkin-Elmer Corp.

Pfizer Inc.

Philips Electronic Instruments Co. (PEI)

Plasma Sciences, Inc.

Plasma-Therm Industrial Products

Plenum Publishing Corp.

PPG Industries Glass R\&D Center

PQ Corporation

The Proctor \& Gamble Company

Prometrix Corporation

Quantum Design, Inc.

Rapro Technology Inc.

Raytheon Company

Resonetics, Inc.

Rhone-Poulenc Inc.

Pigaku USA Inc.

Riken Corporation

Rockwell International Science Center

Sandia National Laboratories

Sanyo Electric Co., Ltd.

Schlumberger-Doll Research

Schlumberger Technologies instruments Division

Scintag, Inc.

Sharp Corporation

Shell Oil Company

Shoei Glass Company, Ltd.

Siemens Analytical X-Ray Instruments, Inc.

Siemens Solar Industries

Sienna Technologies Inc.

Solarex Corporation

Solid State Measurements, Inc.

Sony Co., Ltd.

South Bay Technology, inc.

Spectra-Physics Lasers, Inc.

SpecTran

Spex Industries, Inc.

Spire Corporation

Springer-Verlag New York, Inc.

SSC, Inc.

Staib Instrumente $\mathrm{GmbH}$

Strem Chemicals, Inc.

Sumitomo Electric USA, Inc.

Sumitomo Electric Industries, Ltd.

Sumitomo Metal Mining Co., Ltd.

Superconductive Components, Inc.

Surface/Interface Inc.

Tamarack Scientific Co., Inc.

TDK Corporation

Technics, Inc.

Telios Pharmaceuticals

Texas Instruments, Inc.

Thermionics Laboratory, Inc.

$3 \mathrm{M}$ Company

Toei Industry Co., Lid.

Topcon Technologies, Inc.

Toshiba R\&D Center

Tosoh USA, Inc.

Toyobo Co., Ltd

Ultra High Vacuum Instruments Inc.

Ultratherm Inc.

ULVAC Japan, Ltd.

United Technologies Research Center 


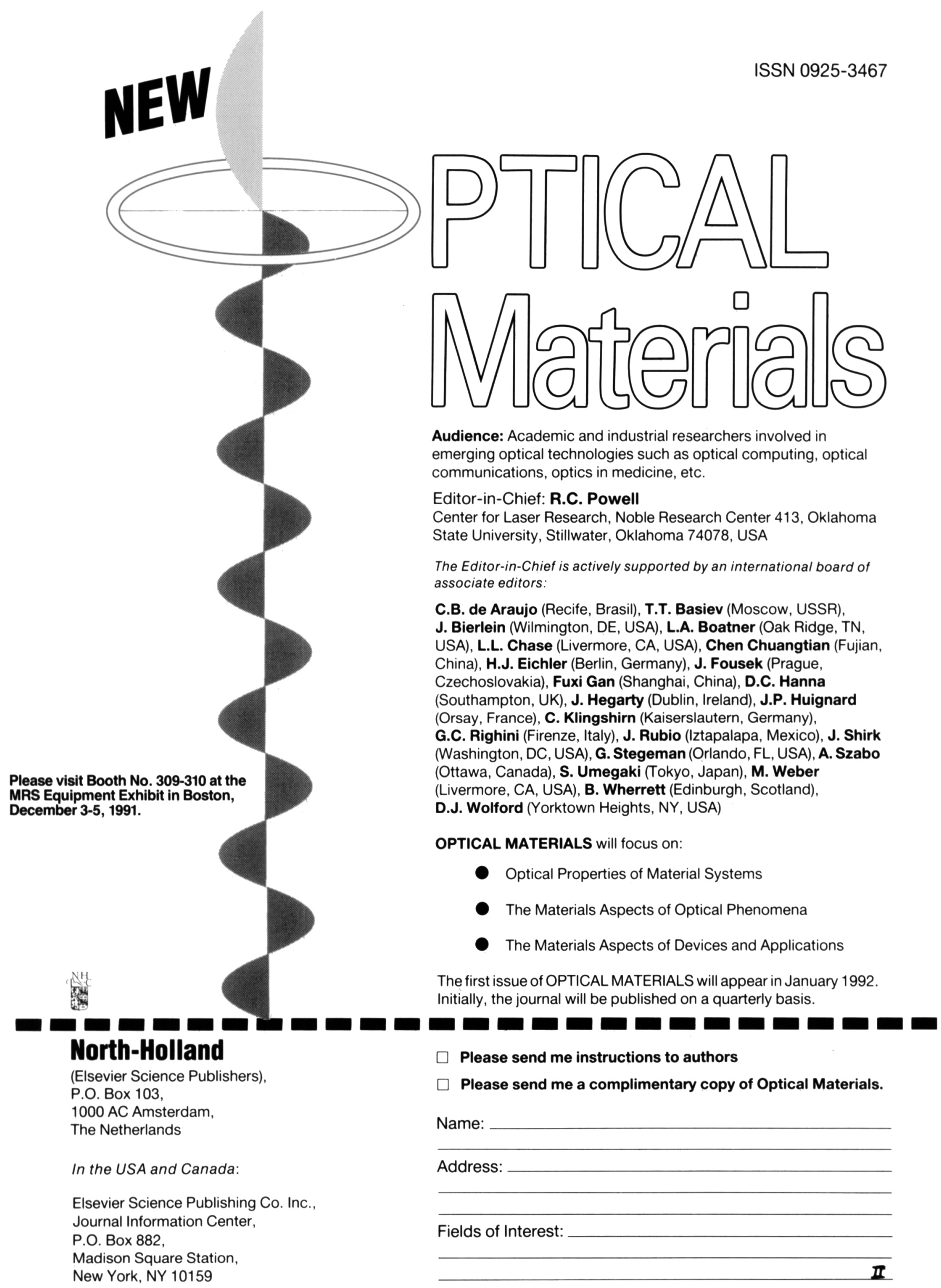

Circle No. 41 on Reader Service Card. 
Please visit Booth No. 610 at the MRS Equipment Exhibit in Boston, December 3-5, 1991.

\section{כ开A}

VASE FTIR MODEL 3

AUTOMATED

VARIABLE ANGLE

SPECTROSCOPIC ELLIPSOMETRY BY FOURRIER TRANSFORM INFRA - RED

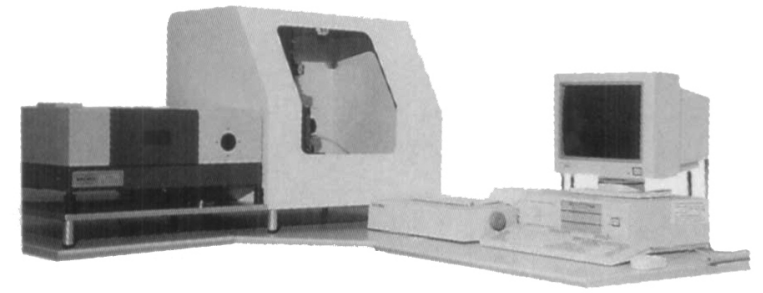

Circle No. 42 on Reader Service Card.
Full FTIR capabilities

Absorbance, transmitance or reflectiance

Advanced computer control \& data analysis

Spectral range: $\quad 5000 \mathrm{~cm}-1$ to $600 \mathrm{~cm}-1(2$ to $16 \mu \mathrm{m})$

Spectral resolution: variable from 2 to $32 \mathrm{~cm}-1$

Beam diameter : $\quad 3$ to $12 \mathrm{~mm}$ manual serring

Angle of incidence : computer controlled, setrable

from $40^{\circ}$ ro $90^{\circ} \pm 0.01^{\circ}$

Standard software package includes :

- System calibration software

- File data hanldling utility software

- Data acquisition and display

- Bulk sample real and imaginary indices calcularion

- Single layer thickness and real and imaginary

index calculation

- Multi layer data reduction

Measurement time : 2 to $420 \mathrm{mn}$

Repeatability : for Tan Psi $=1: \pm 0.006$

for $\operatorname{Cos} \mathrm{Del}=0.5: \pm 0.009$

FOR FURTHER INFORMATION CALL. US AT :

- SOPRA Inc. : 33 NAGOG PARK - PO BOX 2619. ACTOM MA 01720 - 6619 USA

Tel : 5082632520 - Fax : 5082632790

- SOPRA GmbH : Schubertstrasse 9-11

DW 6087 BUETTELBORN - RFA

Tel : 61525092 - Fax : 615255201

- SOPRA S.A. : 26, rue P. Joigneaux - F 92270 Bois Colombes

Tel : 47810949 - Fax : 42422934

o SEIKA Corp : Tel : 333468311 - Fax : 333468314

The M.O.S.S. (Multilayer Optical Scanning Spectrometer) is the latest solution to nondestructive evaluation of multilayer struc. tures in both semiconductor and optical applications. The MOSS offers a number of unique advantages to fine-tune your process :

The solution for

non-destructive evaluation of multilayer structures

Please visit Booth No. 610 at the MRS Equipment Exhibit in Boston, December 3-5, 1991.

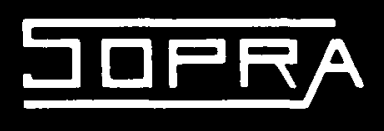

Circle No. 43 on Reader Service Card. 